\title{
Challenges for psychosocial interventions in pediatric oncology
}
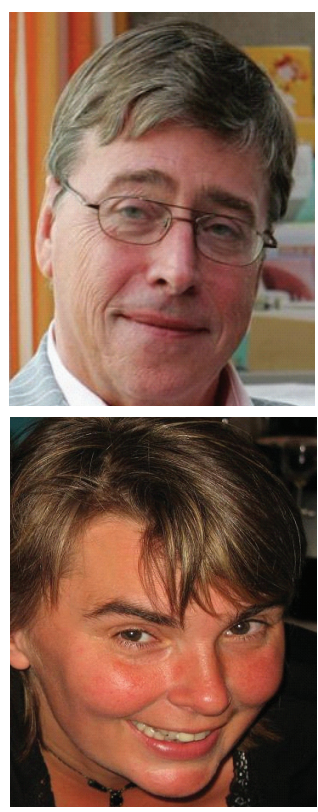

Bob F Last ${ }^{1,2+}$ \& Martha A Grootenhuis ${ }^{3}$

${ }^{\dagger}$ Author for correspondence ${ }^{1}$ Emma Children's Hospital, Pediatric Psychosocial Department, University of Amsterdam, PO Box 22700 1100 DE, Amsterdam, The Netherlands ${ }^{2} V U$ University, Developmental Psychology, Amsterdam,

The Netherlands Tel.: +312056 5674; Fax: +31 2060 91242; E-mail:b.f.last@amc.nl ${ }^{3}$ Principal Investigator, Psychosocial Department, Emma Children's Hospital, Academic Medical Centre, Amsterdam, The Netherlands

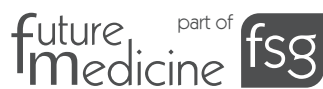

\author{
'... after traveling so often for \\ medical treatment, a substantial \\ number of parents are \\ reluctant to organize \\ additional visits for specialized \\ psychosocial interventions.'
}

Survival rates of children with cancer have increased drastically over the last decades to approximately $70-80 \%$. The therapeutic successes achieved are a result of advances in neuroimaging, surgical techniques and radiotherapy, and induction of newer chemotherapeutic regimens. If $75 \%$ of children with cancer survive their illness, approximately one in every 1000 adults will be a long-term survivor. The burden of cancer, treatment, hospitalization and long-term medical sequela interfere with the children's social and emotional functioning and development.

\section{What is known: psychosocial \& neurocognitive consequences of childhood cancer}

Consequences for pediatric oncology patients have been described extensively. Most research has focused on the psychological consequences and on the neurocognitive morbidity of children with a brain tumor. Although overall mean adjustment has been found to be near normal levels, evidence suggests that more subtle or specific areas may be adversely affected in children with cancer and survivors [1]. Neurocognitive long-term consequences as a result of the treatment for childhood cancer are severe and long lasting and especially affect children treated for brain tumors and children treated with high-dose chemotherapy [2]. Although research confirming educational problems, attentional deficits and working memory deficits in pediatric oncology survivors is well known, empirically validated interventions for these deficits are still scarce. All authors suggest that long-term follow-up of childhood cancer survivors is required to detect and subsequently offer treatment and interventions focused on the cognitive impairments as well as the behavioral and social consequences.
Considering the adverse effects of many treatments, the uncertainty about the further course of the disease and other psychosocial implications of childhood cancer, there is a the need for psychoeducational interventions supporting social and emotional functioning and the need for improvement of (neuro)cognitive functioning in survivors of childhood cancer.

\section{What is offered: interventions in pediatric psycho-oncology}

In pediatric oncology, most interventions are focused on the emotional and social functioning of children with cancer. Interventions related to procedural pain and distress have the strongest empirical support $[3,4]$. It is concluded that evidencebased interventions during and after treatment that address cancer-specific sequela are important [5]. One such promising intervention recently shown to be effective is the program Op Koers [5]. This is a generic psychoeducational program for children with a chronic disease that can be applied within disease-specific groups. First results were positive for children with cancer. Only a few intervention studies have focused on attention and memory problems of brain tumor survivors [6]. Recently, Butler and Mulhern have emphasized that interventions should be developed to improve (neuro)cognitive functioning, thus subsequently improving future perspectives of these children [2].

'Only a few intervention studies have focused on attention and memory problems of brain tumor survivors.'

Psychosocial interventions with the aim to strengthen the child and the family in coping with these late effects have shown their value (e.g., counseling, group interventions, fellow patient/parent meetings and summer camps) but all show logistical problems. Only a small percentage of children and parents take part in these programmes owing to the distance between their home and the treatment center. Moreover, after traveling so often for medical treatment, a substantial number of parents are reluctant to organize additional visits for specialized psychosocial interventions. 


\section{What is new?}

Over the last few years, new interventions have been described. In this editorial we highlight a few examples. There are many indications that survivors with cancer-related brain injury can benefit from cognitive remediation. Recently, neurofeedback had been successfully used with children with attention-deficit hyperactivity disorder $[7,8]$. The underlying assumption of neurofeedback is that brain activity is sensitive to operant conditioning. Several studies have shown a relationship between eletroencephalogram outcomes and operant conditioning procedures [9]. Based on these findings, we assume that childhood cancer survivors suffering from attention and memory problems can also profit from neurofeedback training procedures.

\section{'Development and implementation of intervention programs aimed at empowerment of childhood cancer survivors should be encouraged ... accessibility of these programs has to be enlarged and the internet and web-based interventions are the ultimate place for certain programs.'}

In The Netherlands, a preventive group intervention for young people with depressive symptoms has been developed that is accessed via the internet [101]. A group intervention in a secure internet chat room is provided and was evaluated positively [10]. Another new upcoming approach are web-based interventions. Wade et al. have shown the first beneficial results of a computer-based intervention for parents and children following traumatic brain injury in children [11].

\section{What needs to be done?}

Development and implementation of intervention programs aimed at empowerment of childhood cancer survivors should be encouraged. The accessibility of these programs has to be enlarged and the internet and web-based interventions are the ultimate place for certain programs. Therefore, standardized group intervention programs, including psychoeducation and strengthening of coping abilities, should be adapted and made computer-accessible for the child with cancer and their parents at home. Such interventions could also be appropriate for siblings of children with cancer. Children and parents are able to benefit from interventions delivered via computer on the worldwide web, which eliminate barriers to treatment such as time and distance. In collaboration with The Netherlands' Institute of Mental Health and Addiction (Trimbos Institute), we are in the process of developing the first online course for adolescents with cancer. Furthermore, we believe it is of utmost importance to determine whether neurofeedback is of potential value for pediatric cancer survivors with neurocognitive problems. For this reason we implemented the first pilot study including children and adolescents after treatment for a brain tumor and for those who suffer from substantial attention and/or memory problems.

During and after treatment, the computer and internet can contribute to the adaptation and screening of psychological problems in children with cancer. Computer-assisted assessment has several advantages, including instant data entry and automatic report production of, for example, Health Related Quality of Life (HRQoL) reports. Equivalence of HRQoL measurement properties between paper and pencil methods and electronic modes of administration has been demonstrated. Immediate HRQoL feedback in clinical practice has shown positive effects in adult cancer patients, including improvement of doctor-patient communication, higher levels of patient satisfaction and better identification of 'hidden morbidities' revealed in higher rates of detection of psychological and functional impairments [12].

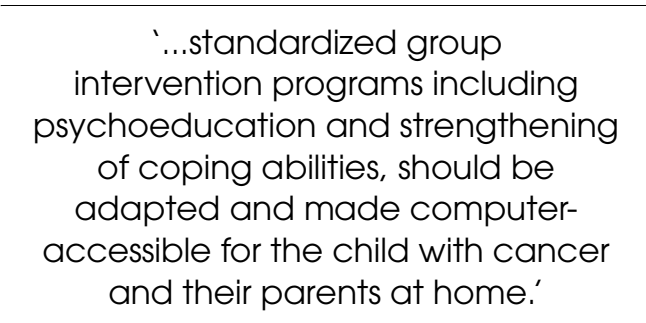

The Quality of Life In Childhood Oncology (QLIC-ON) study, providing patient-reported outcomes to pediatric oncologists is, therefore, currently in progress in our institute. In line with Varni, we encourage the introduction of immediate feedback of HRQoL reports of children and parents in clinical practice, with the aim to improve child-parent-doctors' communication and decision-making during and after treatment. 


\section{Challenges for psychosocial interventions in pediatric oncology - EDITORIAL}

\section{Financial \& competing interests disclosure}

The authors have no relevant affiliations or financial involvement with any organization or entity with a financial interest in or financial conflict with the subject matter or materials discussed in the manuscript. This includes employment, consultancies, honoraria, stock ownership or options, expert testimony, grants or patents received or pending, or royalties.

No writing assistance was utilized in the production of this manuscript.

\section{Bibliography}

1. Patenaude AF, Kupst MJ: Psychosocial functioning in pediatric cancer. J. Pediatr. Psychol. 30(1), 9-27 (2005).

2. Butler RW, Mulhern RK: Neurocognitive interventions for children and adolescents surviving cancer. J. Pediatr. Psychol. 30(1), 65-78 (2005).

3. Pai AL, Drotar D, Zebracki K, Moore M, Youngstrom E: A meta-analysis of the effects of psychological interventions in pediatric oncology on outcomes of psychological distress and adjustment. J. Pediatr. Psychol. 31(9), 978-988 (2006).

4. Kazak AE: Evidence-based interventions for survivors of childhood cancer and their families. J. Pediatr. Psychol. 30(1), 29-39 (2005).

5. Last BF, Stam H,

Onland-van Nieuwenhuizen AM,

Grootenhuis MA: Positive effects of a psycho-educational group intervention for children with a chronic disease: first results. Patient Educ. Couns. 65(1), 101-12 (2007).

6. Barakat LP, Foley B, Carey ME, Gyato K, Phillips PC: Evaluation of a social-skills training group intervention with children treated for brain tumors. A pilot study. J. Pediatr. Psychol. 28(5), 299-307 (2003).

7. Heinrich H, Gevensleben H, Strehl U: Annotation: neurofeedback - train your brain to train behaviour. J. Child Psychol. Psychiatry 48(1), 3-16 (2007).

8. Rossiter $\mathrm{T}$ : The effectiveness of neurofeedback and stimulant drugs in treating ADHD. Part 1. Review of methodological issues. Appl. Psychophysiol. Biofeedback 29(2), 95-112 (2004).

9. Thatcher RW: EEG operant conditioning (biofeedback) and traumatic brain injury. Clin. Electroencephalogr. 31, 38-44 (2000).
10. Gerrits RS, van der Zanden RAP, Visscher RFM, Conijn BP: Master your mood online: a preventive chat group intervention for adolescents. AeJAMH 6(3), 1446-7984 (2007).

11. Wade SL, Wolfe C, Brown TM, Pestian JP: Putting the pieces together: preliminary efficacy of a web-based familyintervention for children with traumatic brain injury. J. Pediatr Psychol. 30(5), 437-442 (2005).

12. Varni JW, Burwinkle TM, Lane MM: Health-related quality of life measurement in pediatric clinical practice: an appraisal and precept for future research and application. Health Qual. Life Outcomes 3, 34 (2005).

\section{Website}

101. Grip op je dip website (The Netherlands) www.gripopjedip.nl 